\title{
A Simple Method for Estimating Activation Energies Using the Fragmentation Yield: Collision-Induced Dissociation of Iron(II)- Phenanthroline Complexes in an Electrospray Ionization Mass Spectrometer
}

\author{
Sandor Keki, Lajos Nagy, Janos Torok, Gyorgy Deak, and Miklos Zsuga \\ Department of Applied Chemistry, University of Debrecen, Debrecen, Hungary
}

\begin{abstract}
The gas-phase stabilities of $\mathrm{Fe}(\Phi)_{3}^{2+}$ complexes, where $\Phi$ represents the 1,10-phenanthroline, 5-chloro-1,10-phenanthroline, 5-methyl-1,10-phenanthroline, 3,4,7,8-tetramethyl-1,10-phenanthroline, and 4,7-diphenyl-1,10-phenanthroline ligands were investigated by collision-induced dissociation (CID) in the capillary-first skimmer region upon changing the voltage difference between the capillary and the skimmer. The loss of only one ligand from the $\mathrm{Fe}(\Phi)_{3}^{2+}$ complexes was observed with each of the phenanthroline ligands studied. An increase in the voltage difference between the capillary and the skimmer resulted in a higher fragmentation yield as calculated from the intensity of the precursor and the fragment ion. The fragmentation yield versus capillary-skimmer voltage difference plots were evaluated by means of the Arrhenius and the Rice-Ramsperger-Kassel (RRK) model by fitting the model parameters to the experimental data. Both models yielded practically the same results. In addition, if the internal energy gained through the capillary-skimmer region is estimated correctly, the approximate value of the critical energy (activation energy) for fragmentation can be extracted from the fragmentation yield versus capillary-skimmer voltage difference plots. It was found that the gas-phase stabilities of the $\mathrm{Fe}(\Phi)_{3}^{2+}$ complexes are nearly identical except for the more stable Fe(II)-4,7-diphenyl-1,10-phenanthroline complex. The critical energy for fragmentation was estimated to be $\sim 1.2$ and $0.9 \mathrm{eV}$ for the Fe(II)- 4,7-diphenyl-1,10-phenanthroline, and the other complexes, respectively. (J Am Soc Mass Spectrom 2006, 17, 962-966) @ 2006 American Society for Mass Spectrometry
\end{abstract}

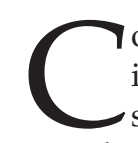
omparison of the stabilities of the metal-containing complexes in the gas-phase to those in the solution phase may provide a deeper insight into the processes affecting the complex formation. The electrospray ionization (ESI) technique [1] combined with tandem mass spectrometry offers a unique way for the investigation of the metal-containing complexes by generating gas-phase ions from solutions under the influence of a constant electric field [2-8]. The gasphase ions formed in such a way are then mass analyzed and/or mass-selected, followed by collision with the collision gas molecules (CID) in the collision cell or surface (SID) to induce fragmentation and, thus, structural information and bond dissociation energies for the analyte ions can be obtained. Several methods have been applied for determination of relative gas-phase

Published online May 19, 2006

Address reprint requests to Dr. M. Zsuga, Department of Applied Chemistry, University of Debrecen, H-4010 Debrecen, Hungary. E-mail: zsugam@ tigris.klte.hu stabilities and critical energy for fragmentation including threshold dissociation voltage [6], critical slope method [7], sigmoid-type evaluation [9], as well as a highly sophisticated computer program based on the Rice-Ramsperger-Kassel-Marcus (RRKM) approximation is also available [10].

In the most commercial ESI-MS instruments with no MS/MS capabilities, fragmentation can only be enhanced upon increasing the potential difference between the orifice (capillary) and the skimmer. The ions entering from the atmospheric pressure region into the first stage of the vacuum system are accelerated by the orifice-skimmer potential difference, meanwhile several low and/or high-energy collisions occur between the ions and the background gas molecules. Due to the collisions and the accompanied kinetic to internal energy-transfer, the ion internal energy increases as it passes through the orifice-skimmer region thereby increasing the possibility of fragmentation. The increase in the internal energy gained form multiple collisions in this region is proportional to the orifice-skimmer voltage 
difference, i.e., the amount of internal energy deposited to the ions can be controlled [11, 12].

In this article, we report the determination of the gas-phase stabilities of five 1,10-phenanthroline derivatives complexed with $\mathrm{Fe}^{2+}$ ions $\left[\mathrm{Fe}(\Phi)_{3}^{2+}\right]$ using collision-induced dissociation (CID) in the capillary-first skimmer region of a single ESI-MS instrument. Phenanthroline ligands were selected for this study because they are among the most utilized chelating agents in coordination chemistry forming stable complexes with $\mathrm{Fe}^{2+}$ ions in solution [13-17]. In addition, gas-phase stabilities of the $\mathrm{Fe}(\Phi)_{3}^{2+}$ complexes are less-known.

\section{Experimental}

\section{Materials}

1,10-phenanthroline, 5-chlor-1,10-phenanthroline, 5-methyl-1,10-phenanthroline, 3,4,7,8-tetramethlyl-1,10-phenanthroline, and 4,7-diphenyl-1,10-phenanthroline (bathophenanthroline), $\mathrm{FeSO}_{4} 7 \mathrm{H}_{2} \mathrm{O}$, and ethanol were received from Aldrich (Seelze, Germany). The stock solutions of $\mathrm{Fe}(\mathrm{II})$-phenanthroline complexes were prepared at a concentration of $10^{-3} \mathrm{M}$ in water (the Fe(II)-bathophenanthroline complex was prepared in ethanol) with 1:3 $\mathrm{M}$ ratio [Fe(II)/ligand].

\section{Electrospray Ionization Time-of-Flight Mass Spectrometry (ESI-TOF MS)}

ESI-TOF MS measurements were performed with a BioTOF II instrument (Bruker Daltonics, Billerica, MA). The concentration of the Fe(II)-phenanthroline complexes was $10 \mu \mathrm{M}$. The solutions were introduced directly into the ESI source with a syringe pump (Cole-Parmer Ins. Comp., Vermont Hills, IL) at a flow rate of $2 \mu \mathrm{L} / \mathrm{min}$. The temperature of the drying gas $\left(\mathrm{N}_{2}\right)$ was maintained at $100^{\circ} \mathrm{C}$. The voltages applied on the capillary entrance, and the second skimmer were $-4500 \mathrm{~V}$ and $30 \mathrm{~V}$, respectively. The voltage on the first skimmer was kept at $40 \mathrm{~V}$, while the voltage on the capillary exit was varied from 45 to $220 \mathrm{~V}$. To induce fragmentation of the Fe(II)-complexes the voltage on the capillary exit was increased gradually while keeping the first skimmer voltage constant. The spectra at different capillary-skimmer differences were accumulated and recorded by a digitizer at a sampling rate of $2 \mathrm{GHz}$.

\section{Parameter Estimation}

For fitting the parameters of the Arrhenius and RRK models to the experimental fragmentation curves, a home-made computer program written in Turbo Pascal using the Gauss-Newton-Marquardt procedure was employed [18].

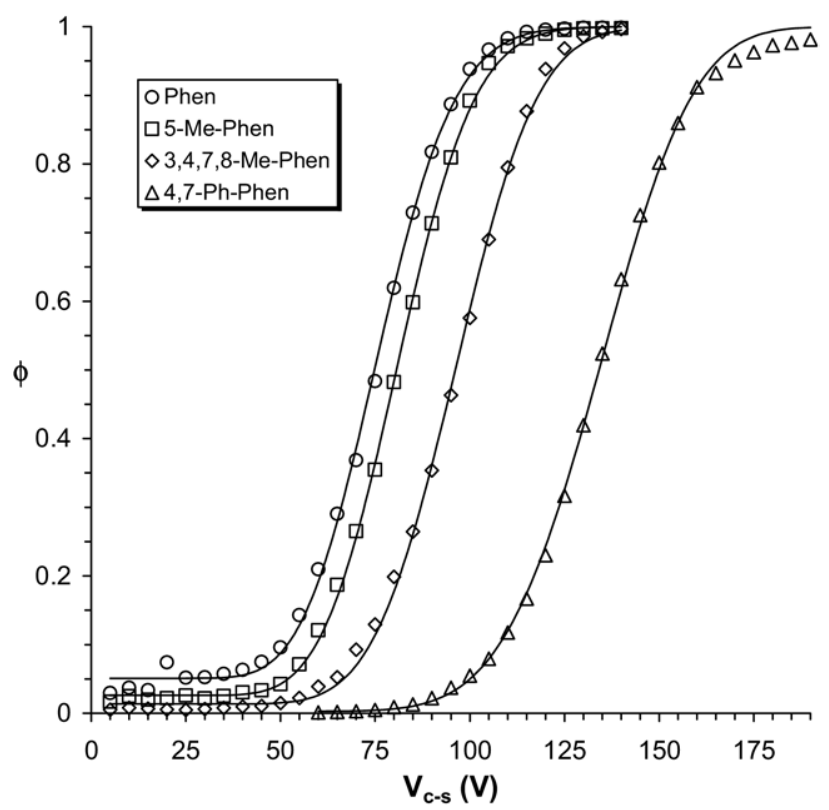

Figure 1. Fragmentation yield $(\phi)$ versus $V_{c-s}$ plots for the $\mathrm{Fe}(\Phi)_{3}^{2+}$ complexes. The solid lines represent the fitted curves with eq 7 .

\section{Results and Discussion}

The solutions of the 1,10-phenanthroline ligands $(\Phi)$ complexed with $\mathrm{Fe}^{2+}$ ions as $\mathrm{Fe}(\Phi)_{3}^{2+}$ were directly injected into the ESI source at varying capillary-skimmer potential differences.

Upon increasing the potential difference, fragmentation of $\mathrm{Fe}(\Phi)_{3}^{2+}$ to $\mathrm{Fe}(\Phi)_{2}^{2+}$ becomes more pronounced. It should be noted that the loss of only one ligand from the $\mathrm{Fe}(\Phi)_{3}^{2+}$ tris-complexes was observed in each case. The fragmentation yields $(\phi)$ were calculated according to eq 1 .

$$
\phi=\mathrm{I}_{\mathrm{B}} /\left(\mathrm{I}_{\mathrm{T}}+\mathrm{I}_{\mathrm{B}}\right)
$$

where $I_{T}$ and $I_{B}$ represent the intensities of the tris(precursor) and bis-complex, respectively.

The fragmentation yields are plotted as a function of the potential difference between the capillary and the skimmer and these plots are shown in Figure 1, which indicates that each $\mathrm{Fe}(\Phi)_{3}^{2+}$ complex produces a sigmoid-like fragmentation curve. Non-neglible decays were observed even at low capillary-skimmer voltage differences $\left(\mathrm{V}_{\mathrm{c}-\mathrm{s}}\right)$. On the other hand, it is also evident from Figure 1 that decays at low $\mathrm{V}_{\mathrm{c}-\mathrm{s}}$ decrease in the order of Phen $\approx 5$-Cl-Phen $>5$-Me-Phen $>3,4,7,8$-MePhen $>4,7-\mathrm{Ph}-\mathrm{Phen}$. In the case of 4,7-Ph-Phen practically no fragmentation occurs at low $\mathrm{V}_{\mathrm{c}-\mathrm{s}}$. The observed decays at low $\mathrm{V}_{\mathrm{c}-\mathrm{s}}$ can be attributed to the fragmentation of the $\mathrm{Fe}(\Phi)_{3}^{2+}$ complex before entering into the capillary-skimmer region and/or dissociation in the RF-only hexapole after the skimmer. We do believe that the former assumption is more probable than the latter, since in the hexapole collisional cooling, rather than 
Table 1. The values of the fitted parameters of the Arrhenius and RRK model. (The calculated errors are shown in parenthesis)

\begin{tabular}{|c|c|c|c|c|c|c|}
\hline & \multicolumn{3}{|c|}{ Arrhenius } & \multicolumn{3}{|c|}{ RRK } \\
\hline & a & $\mathrm{b}$ & c & a & $\mathrm{b}$ & c \\
\hline Phen & $5.21 \times 10^{-2}\left(3.62 \times 10^{-3}\right)$ & $167.3(16.4)$ & $2.13(0.04)$ & $5.23 \times 10^{-2}\left(3.7 \times 10^{-3}\right)$ & $155.9(15.2)$ & $2.09(0.04)$ \\
\hline 5-Cl-Phen & $3.75 \times 10^{-2}\left(2.7 \times 10^{-3}\right)$ & $147.9(10.4)$ & $2.09(0.03)$ & $3.8 \times 10^{-2}\left(2.87 \times 10^{-3}\right)$ & $138.2(9.7)$ & $2.04(0.03)$ \\
\hline 5-Me-Phen & $2.62 \times 10^{-2}\left(2.4 \times 10^{-3}\right)$ & $250.8(18.6)$ & $2.15(0.03)$ & $2.65 \times 10^{-2}\left(2.5 \times 10^{-3}\right)$ & $233.3(17.4)$ & $2.11(0.03)$ \\
\hline 3,4,7,8-Me-Phen & $1.36 \times 10^{-2}\left(3.2 \times 10^{-3}\right)$ & $467.3(55.1)$ & $2.07(0.04)$ & $1.37 \times 10^{-2}\left(3.2 \times 10^{-3}\right)$ & $437.3(51.5)$ & $2.03(0.04)$ \\
\hline 4,7-Ph-Phen & $2.74 \times 10^{-3 *}\left(4.3 \times 10^{-3}\right)^{*}$ & $1630(240)$ & $2.78(0.05)$ & $2.97 \times 10^{-3 *}\left(4.2 \times 10^{-3}\right)^{*}$ & $1510(216)$ & $2.73(0.05)$ \\
\hline
\end{tabular}

*The value of $a$ can be regarded practically zero due to its low value and the associated large calculated error.

heating occurs as pointed out in some studies [11, 12]. To describe the fragmentation curves of the $\mathrm{Fe}(\Phi)_{3}^{2+}$ complexes within the framework of unimolecular dissociation, let the rate constant of unimolecular dissociation be $\mathrm{k}_{\mathrm{o}}$ and the time taken for the ions to travel this region before expansion into the capillary end-skimmer region be $\tau_{\mathrm{o}}$, then the number of the undissociated ions can be expressed by eq 2 .

$$
\mathrm{P}=\mathrm{P}_{\mathrm{O}} e^{-\mathrm{k}_{\mathrm{o}} \tau_{\mathrm{O}}}
$$

where $\mathrm{P}_{\mathrm{o}}$ is the initial number of the undissociated ions and $\mathrm{P}$ is the number of the undissociated ions at time $\tau_{\mathrm{o}}$.

In the capillary-skimmer region the ions gain additional internal energy due to the collision with the background gas molecules, and the average unimolecular rate constant for this region is taken to be $\mathrm{k}$. The number of undissociated ions reaching the detector within the time $\tau$ is given by eq 3 .

$$
\mathrm{P}=\mathrm{P}_{\mathrm{O}} e^{-\mathrm{k}_{\mathrm{o}} \tau_{\mathrm{o}}-\mathrm{k} \tau}
$$

The fraction of the dissociated $\mathrm{Fe}(\Phi)_{3}^{2+}$ complex, i.e., the fragmentation yield is then expressed by eq 4 .

$$
\phi=1-\exp \left[-\mathrm{k}_{\mathrm{o}} \tau_{\mathrm{o}}-\mathrm{k} \tau\right]
$$

It is supposed that the internal energy acquired before the capillary-skimmer region is $\mathrm{E}_{\mathrm{int}, \mathrm{o}}$ and an additional $E_{\text {int }}$ internal energy is accumulated within this region. The initial effective temperature before entering the capillary-skimmer region and within the region is approximated by eq 5 .

$$
\mathrm{E}_{\text {int }, o}=\mathrm{sRT}_{\mathrm{o}} \text { and } \mathrm{E}_{\text {int }}+\mathrm{E}_{\text {int, o }}=\mathrm{sRT}_{\text {eff }}
$$

where $\mathrm{s}$ is the degree of freedom (DOF) and $\mathrm{R}$ being the gas constant, $\mathrm{T}_{\mathrm{o}}$ and $\mathrm{T}_{\text {eff }}$ are the "effective temperatures".

Using eq 5 for $k_{o}$ and $k$ the Arrhenius equation is read as:

$$
\begin{aligned}
& \mathrm{k}_{\mathrm{o}}=\operatorname{Aexp}\left(-\mathrm{sE}_{\mathrm{a}} / \mathrm{E}_{\text {int }, \mathrm{o}}\right) \text { and } \\
& \mathrm{k}=\operatorname{Aexp}\left[-\mathrm{sE}_{\mathrm{a}} /\left(\mathrm{E}_{\text {int }, \mathrm{o}}+\mathrm{E}_{\text {int }}\right)\right]
\end{aligned}
$$

Assuming that $E_{\text {int }} \gg E_{\text {int,o }}$ and substituting eq 6 into eq 4 the fragmentation yield according to the Arrhenius equation $\left(\phi_{\mathrm{ARR}}\right)$ is given by eq 7 .

$$
\begin{aligned}
\phi_{\text {ARR }}\left(\mathrm{E}_{\text {int }}\right)= & 1-\exp \left[-\mathrm{A} \tau_{\mathrm{o}} \exp \left(-\mathrm{sE}_{\mathrm{a}} / \mathrm{E}_{\text {int, } \mathrm{o}}\right)\right. \\
& \left.-\mathrm{A} \tau \exp \left(-\mathrm{sE}_{\mathrm{a}} / \mathrm{E}_{\text {int }}\right)\right]
\end{aligned}
$$

Supposing that the internal energy gained in this region is proportional to the applied potential difference, i.e., $\mathrm{E}_{\mathrm{int}}=\alpha \mathrm{V}_{\mathrm{c}-\mathrm{s}}$, where $\alpha$ is the proportionality constant, the following working function can be constructed:

$$
\phi_{\text {ARR }}\left(\mathrm{V}_{\mathrm{c}-\mathrm{s}}\right)=1-\exp \left[-a-b \exp \left(-\mathrm{s} \times c / \mathrm{V}_{\mathrm{c}-\mathrm{s}}\right)\right]
$$

where $a, b$, and $c$ are constants: $a=\mathrm{A} \tau_{\mathrm{o}} \exp \left(-\mathrm{sE}_{\mathrm{a}} / \mathrm{E}_{\mathrm{int}, \mathrm{o}}\right)$, $b=\mathrm{A} \tau$ and $c=\mathrm{E}_{\mathrm{a}} / \alpha$.

In the case of RRK approximation, $\mathrm{k}_{\mathrm{o}}$ and $\mathrm{k}$ is expressed by eq 9 :

$$
\begin{aligned}
& \mathrm{k}_{\mathrm{o}}=\mathrm{A}\left(1-\mathrm{E}_{\mathrm{o}} / \mathrm{E}_{\text {int }, \mathrm{o}}\right)^{\mathrm{S}-1} \text { and } \\
& \mathrm{k}=\mathrm{A}\left[1-\mathrm{E}_{\mathrm{o}} /\left(\mathrm{E}_{\text {int }, \mathrm{o}}+\mathrm{E}_{\text {int }}\right)\right]^{\mathrm{s}-1}
\end{aligned}
$$

where $E_{o}$ stands for the critical energy for fragmentation.

Similarly, by substituting eq 9 into eq 4 and assuming that $\mathrm{E}_{\text {int }} \gg \mathrm{E}_{\text {int,o, }}$, the fragmentation yield according to the RRK model $\left(\phi_{\mathrm{RRK}}\right)$ can be given with eq 10 .

$$
\begin{aligned}
\phi_{\text {RRK }}\left(\mathrm{E}_{\text {int }}\right)= & 1-\exp \left[-\mathrm{A} \tau_{\mathrm{o}}\left(1-\mathrm{E}_{\mathrm{o}} / \mathrm{E}_{\text {int }, \mathrm{o}}\right)^{\mathrm{s}-1}\right. \\
& \left.-\mathrm{A} \tau\left(1-\mathrm{E}_{\mathrm{o}} / \mathrm{E}_{\text {int }}\right)^{\mathrm{s}-1}\right]
\end{aligned}
$$

and the working function is expressed by eq 11 .

$$
\phi_{\mathrm{RRK}}\left(\mathrm{V}_{\mathrm{c}-\mathrm{s}}\right)=1-\exp \left[-a-b\left(1-c / \mathrm{V}_{\mathrm{c}-\mathrm{s}}\right)^{\mathrm{s}-1}\right]
$$

where $a=\mathrm{A} \tau_{\mathrm{o}}\left(1-\mathrm{E}_{\mathrm{o}} / \mathrm{E}_{\mathrm{int}, \mathrm{o}}\right)^{\mathrm{s}-1}, b=\mathrm{A} \tau$, and $c=\mathrm{E}_{\mathrm{o}} / \alpha$.

The parameters $a, b$, and $c$ of eq 8 and eq 11 were estimated by fitting these equations to the experimental $\phi$ values. Examples of such fits are shown in Figure 1 and the fitted values of $a, b$, and $c$ for the Arrhenius and the RRK models are summarized in Table 1.

As it turns out from Figure 1, the calculated curves fit well to the experimental data. Also, the values of $a, b$, and $c$ are practically the same for both the Arrhenius and the RRK model. The reason for this similarity is that the Arrhenius approximation can be regarded as a particular case of the RRK model as outlined in the Appendix.

To determine the value of $\mathrm{E}_{\mathrm{o}}$, the value of $\alpha$ should be known. For the estimation of $\alpha$, model calculations 


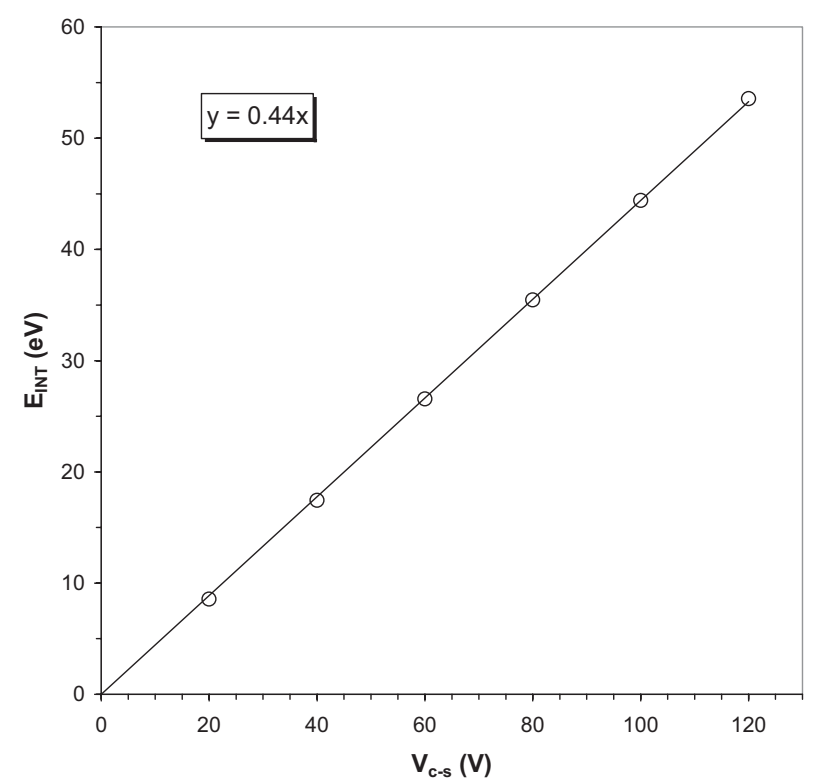

Figure 2. Calculated internal energy for the ferroin ( $\mathrm{Fe}(\mathrm{phen})_{3}^{2+}$ ) complex as a function of $\mathrm{V}_{\mathrm{c}-\mathrm{s}}$ assuming partially inelastic collisions $(\eta=0.5)$ with the background gas.

were performed based on the equations for the free-jet expansion into a vacuum [11]. These equations were applied to our ESI interface using the relevant geometrical dimensions of this source provided by the manufacturer. Briefly, in these calculations the internal energy of the complex ion was considered as the sum of energy transferred from all individual collision events between the capillary and the skimmer. The variations in the gas number density and the mean free path in the capillary-skimmer were taken into account and the total number of collisions of the complex ion with the background gas molecules in this region was calculated using a spreadsheet software. In these calculations we considered partially inelastic collisions and the kinetic energy transferred to internal energy of the ion in an individual collision event $\left(\Delta \mathrm{E}_{\mathrm{int}}\right)$ was taken as:

$$
\begin{aligned}
& \mathrm{E}_{\mathrm{CM}}=\mathrm{m}_{\mathrm{g}} /\left(\mathrm{m}_{\mathrm{i}}+\mathrm{m}_{\mathrm{g}}\right) \mathrm{E}_{\text {kin }} \\
& \Delta \mathrm{E}_{\text {int }}=\eta \mathrm{E}_{\mathrm{CM}}
\end{aligned}
$$

where $m_{i}$ and $m_{g}$ are the mass of the ion and the background gas, $\mathrm{E}_{\text {kin }}$ is the kinetic energy of the ion before collision, and $\eta$ is the fraction of center of mass energy $\left(\mathrm{E}_{\mathrm{CM}}\right)$ transferred.

The increase in the kinetic energy of the ion $\left(\Delta \mathrm{E}_{\mathrm{kin}}\right)$ between two collisions is:

$$
\Delta \mathrm{E}_{\mathrm{kin}}=\mathrm{q} \varepsilon \lambda
$$

where $q, \varepsilon$, and $\lambda$ are the charge of the ion, the electric field between the capillary and the skimmer and the mean free path, respectively.

The kinetic energy loss due to collision and energytransfer:

$$
\Delta \mathrm{E}_{\text {loss }}=-\mathrm{E}_{\mathrm{CM}}\left(2 \mathrm{~m}_{\mathrm{i}}+\eta \mathrm{m}_{\mathrm{g}}\right) /\left(\mathrm{m}_{\mathrm{i}}+\mathrm{m}_{\mathrm{g}}\right)
$$

Using eqs 12-15 together with the equations for the free-jet expansion [11], the internal energy input as a function of the capillary-skimmer voltage differences was calculated. The collision cross-sections were estimated using the corresponding bond distances (calculated with the Chemoffice software) and were assumed to be constant through the capillary-skimmer region. The cross-sections used in the calculations were $1.4 \times$ $10^{-14}, 1.6 \times 10^{-14}, 1.8 \times 10^{-14}, 1.9 \times 10^{-14}$, and $2.6 \times$ $10^{-14} \mathrm{~cm}^{2}$ for the Phen, 5-Cl-Phen, 5-Me-Phen, 3,4,7,8Me-Phen, and 4,7-Ph-Phen $\mathrm{Fe}(\Phi)_{3}^{2+}$ complexes, respectively. The internal energy input versus $V_{c-s}$ plot for the ferroin complex assuming partially inelastic collision with $\eta=0.5$ is shown in Figure 2 .

Figure 2 shows a linear relationship between $\mathrm{E}_{\text {int }}$ and $\mathrm{V}_{\mathrm{c}-\mathrm{s}}$ with a slope of $\alpha=0.44 \mathrm{eV} / \mathrm{V}$. Interestingly, practically the same value of $\alpha$ was obtained for the other $\mathrm{Fe}(\Phi)_{3}^{2+}$ complexes. The reason for this is that with increasing mass of the complex ion the fraction of the kinetic energy converted to internal energy decreases. On the other hand, the cross-section also increases with the mass of the complex ion giving rise to an increase in the number of collisions. Since the total internal energy input is the product of the collision number and the fraction of kinetic energy converted to internal energy, these two effects cancel each other resulting in practically the same $\mathrm{E}_{\mathrm{int}}-\mathrm{V}_{\mathrm{c}-\mathrm{s}}$ plot (with the same slope, i.e., $\alpha$ ). Using the data of Table 1, row 3 (values of parameter $c$ ) and considering that the value of $\alpha$ is approximately the same for all of the $\mathrm{Fe}(\Phi)_{3}^{2+}$ complexes (within 3\%), the relative gas-phase stabilities of these complexes are in the following order: 4,7-PhPhen $>$ Phen $\approx 5$-Cl-Phen $\approx 5$-Me-Phen $\approx 3,4,7,8$-MePhen. The gas-phase stabilities of the $\mathrm{Fe}(\Phi)_{3}^{2+}$ complexes are consistent with those in the solution phase except with that of the Fe(4,7-Ph-Phen) ${ }_{3}^{2+}$ complex (Table 2).

The reason for this may be that the electron-donating effect of the phenyl groups in solvent-free environment is more expressed in the gas phase than in solution giving rise to higher stability of the complex in the gas-phase. In addition, the activation energy (critical energy) for fragmentation can be estimated using the data of Table 1 row 3 (values of parameter $c$ ) and the value of $\alpha$. With these data, one can calculate approxi-

Table 2. The values of $\log \beta_{3}$ for the $\mathrm{Fe}(\Phi)_{3}{ }^{2+}$ complexes in aqueous solution [19]

\begin{tabular}{lc}
\hline & $\log \beta_{3}{ }^{*}$ \\
\hline \hline Phen & 21.1 \\
5-Cl-Phen & 19.7 \\
5-Me-Phen & 22.1 \\
3,4,7,8-Me-Phen & unknown \\
4,7-Ph-Phen & $21.8^{* *}$ \\
\hline
\end{tabular}

${ }^{*} \beta_{3}$ is the stability constant, i.e., $\beta_{3}=\left[\mathrm{Fe}(\Phi)_{3}{ }^{2+}\right] /\left(\left[\mathrm{Fe}^{2+}\right][\Phi]^{3}\right)$.

**Determined in ethanol. 
mately a critical energy for the fragmentation of 1.2 and $0.9 \mathrm{eV}$ for the $\mathrm{Fe}(\mathrm{II})-4,7$-diphenyl-1,10-phenanthroline, and the other complexes, respectively. Although the determined critical energies can be regarded as semiquantitative and their values greatly depend on the correct estimation of the fraction of the kinetic to internal energy conversion $(\eta)$, the relative gas-phase stabilities can be easily deduced.

\section{Conclusions}

In this article, we presented the determination of relative gas-phase stabilities of five phenanthroline derivatives complexed with $\mathrm{Fe}^{2+}$ ions. The sigmoid-like fragmentation yield versus capillary voltage differences plots were evaluated using the Arrhenius equation and the RRK model by considering a simple unimolecular kinetics for the dissociation occurring in the capillaryskimmer region of a single ESI-MS instrument. It was found that the Arrhenius and RRK model yielded the same results. The relative gas-phase stabilities of these complexes were in good agreement with those in the solution phase. Although the determined critical energies can be regarded as semi-quantitative, and their values are greatly dependent on the correct estimation of the fraction of the kinetic to internal energy conversion $(\eta)$, the relative gas-phase stabilities can be easily deduced from the method presented in this paper.

\section{Acknowledgments}

This work was financially supported by grants T 037448, T 042740, M 28,369, and M 36,872, MU-00,204/2001 given by OTKA (National Scientific Research Fund, Hungary), grant RET 006/2004 and the Bolyai János Fellowship.

\section{Appendix}

Using the RRK model, i.e., $\mathrm{k}=\mathrm{A}\left(1-\frac{\mathrm{E}_{\mathrm{O}}}{\mathrm{E}_{\text {int }}}\right)^{\mathrm{s}-1}$ and rearranging it to yield $\mathrm{k}=\mathrm{A}\left[1-\frac{1}{\mathrm{~s}-1} \frac{\mathrm{E}_{\mathrm{O}}(\mathrm{s}-1)}{\mathrm{E}_{\text {int }}}\right]^{\mathrm{s}-1}$.

At sufficiently large values of $\mathrm{s}$ it holds that

$$
\begin{aligned}
& \lim \left(1-\frac{1}{s-1}\right)^{s-1}=e^{-1}, \text { i.e., } \mathrm{k}= \\
& \mathrm{A}\left(1-\frac{\mathrm{E}_{\mathrm{O}}}{\mathrm{E}_{\text {int }}}\right)^{\mathrm{s}-1}=\operatorname{Aexp}\left[-\frac{\mathrm{E}_{\mathrm{O}}(\mathrm{s}-1)}{\mathrm{E}_{\text {int }}}\right]
\end{aligned}
$$

\section{References}

1. Wong, S. F.; Meng, C. K.; Fenn, J. B. Multiple charging in electrospray ionization of poly(ethylene glycols). J. Phys. Chem. 1988, 92, 546-550.

2. Vachet, R. W.; Hartman J. R.; Gertner, J. W.; Callahan, J. H. Investigation of metal complex coordination structure using collision-induced dissociation and ion-molecule reactions in a quadrupole ion trap mass spectrometer. Int. J. Mass Spectrom. 2001, 204, 101.

3. Vachet, R. W.; Callahan, J. H. Quadrupole ion trap studies of the structure and reactivity of transition metal ion pair complexes. J. Mass Spectrom. 2000, 35, 311-320.

4. Molina-Svendsen, H.; Bojesen, G.; McKenzie, C. J. Gas-phase reactivity of coordinatively unsaturated transition metal complex ions toward molecular oxygen. Inorg. Chem. 1998, 37 1981-1983.

5. Tsierkezos, N. G.; Diefenbach, M.; Roithova, J.; Schroder, D.; Schwarz, H. Competitive complexation of gaseous Mn-II by 1,10phenanthroline, 2,2'-bipyridine, and 4,5-diazafluorene. Inorg. Chem. 2005, 44, 4969-4978.

6. Satterfield, M.; Brodbelt, J. S. Relative binding energies of gas-phase pyridyl ligand/metal complexes by energy-variable collisionally activated dissociation in a quadrupole ion trap. Inorg. Chem. 2001, 40, 5393-5400.

7. Forbes, M. W.; Volmer, D. A.; Francis, G. J.; Bohme, D. K. A comparison of data analysis methods for determining gas phase stabilities by CID: Alkali metal complexes of polyether ionophore antibiotics. J. Am. Soc. Mass Spectrom. 2005, 16, 779-791.

8. Hayes, L. A.; Chappell, A. M.; Jellen, E. E.; Ryzhov, V. Binding of metalloporphyrins to model nitrogen bases: collision-induced dissociation and ion-molecule reaction studies. Int. J. Mass Spectrom. 2003, 227, $111-120$.

9. Schroder, D.; Engeser, M.; Bronstrup, M.; Daniel, C.; Spandl, J.; Hartl, H. Ion chemistry of the hexanuclear methoxo-oxovanadium cluster V6O7(OCH3)(12). Int. J. Mass Spectrom. 2003, 228, 743-757.

10. Drahos, L.; Vekey, K. Mass Kinetics: A theoretical model of mass spectra incorporating physical processes, reaction kinetics, and mathematical descriptions. J. Mass Spectrom. 2001, 36, 237-263.

11. Schneider, B. B.; Chen, D. D. Y. Collision-induced dissociation of ions within the orifice-skimmer region of an electrospray mass spectrometer. Anal. Chem. 2000, 72, 791-799.

12. Schneider, B. B.; Douglas, D. J.; Chen, D. D. Y. Collision-induced dissociation of bradykinin ions in the interface region of an ESI-MS. J. Am. Soc. Mass Spectrom. 2001, 12, 772-779.

13. Capone, S.; Derobertis, A.; Destefano, C.; Scarcella, R. Thermodynamics of formation of magnesium, calcium, strontium, and barium complexes with 2,2'-bipyridyl and 1,10-phenanthroline at different ionic strengths in aqueous solution. Talanta, 1985, 32, 675-677.

14. Sammes, P. G.; Yahioglu, G. 1,10-Phenanthroline-A versatile ligand. Chem. Soc. Rev. 1994, 23, 327-334.

15. Bandyopadhyay, S.; Mukherjee, G. N.; Drew, M. G. B. Structural and equilibrium studies on mixed ligand complexes of $\mathrm{Co}(\mathrm{II}), \mathrm{Ni}(\mathrm{II}), \mathrm{Cu}(\mathrm{II})$, and $\mathrm{Zn}(\mathrm{II})$ with N-(2-benzimidazolyl)methyliminodiacetic acid and typical N,N donor ligands. Inorg. Chim. Acta 2005, 358, 3786-3798.

16. Ishimori, K.; Imura, H.; Ohashi, K. Effect of 1,10-phenanthroline on the extraction and separation of lithium(I), sodium(I), and potassium(I) with thenoyltrifluoroacetone. Anal. Chim. Acta, 2002, 454, 241-247.

17. Mudasir, Yoshioka, N.; Inoue, H. DNA binding of iron(II) mixed-ligand complexes containing 1,10-phenanthroline and 4,7-diphenyl-1,10phenanthroline. J. Inorg. Biochem. 1999, 77, 239-247.

18. Bard, Y. Nonlinear Parameter Estimation; Academic Press: New York, 1974 , p. 341.

19. Martell, A. E.; Smith, R. M. Critical stability constants, Vol. II. Amines; Plenum Press: New York, 1974/1989. 